\title{
Pancreatic Serous Adenoma
}

National Cancer Institute

\section{Source}

National Cancer Institute. Pancreatic Serous Adenoma. NCI Thesaurus. Code C95470.

A benign, non-metastasizing, usually cystic epithelial neoplasm arising from the exocrine pancreas. It is composed of glycogen-rich epithelial cells which produce a watery fluid. Signs and symptoms include abdominal mass, abdominal pain, nausea, vomiting, and weight loss. 